Annual Conference on Magnetism and Magnetic Materials Atlanta, Georgia, November 11, 1996

$$
\text { CONF-96/141-- } 4
$$

"The submitted manuscript has been authored by a contractor of the US Government under by a contractor of the U.S. Government unde Accordingly, the U.S. Government retains a nonexclusive, royalty-free license to publish or reproduce the published form of this contribution, or allow others to do so, for U.S. Government purposes."

RECEIVED SEP 191996

O \& TI

\title{
CHARGE-DENSITY WAVE AND MAGNETIC PHASE DIAGRAM OF CHROMIUM ALLOYS
}

R. S. Fishman, X. W. Jiang

SOLID STATE DIVISION

OAK RIDGE NATIONAL LABORATORY

Managed by

LOCKHEED MARTIN ENERGY RESEARCH CORP.

Under

Contract No. DE-AC05-96OR22464

With the

U. S. DEPARTMENT OF ENERGY

OAK RIDGE, TENNESSEE

August 1996 


\title{
CHARGE-DENSITY WAVE AND MAGNETIC PHASE DIAGRAM OF CHROMIUM ALLOYS
}

\author{
R.S. Fishman \\ Solid State Division, P.O. Box 2008, Oak Ridge National Lab, Oak Ridge, TN 37831-6032 \\ X.W. Jiang* \\ Physics Department, North Dakota State University, Fargo, ND 58105-5566
}

(August 8, 1996)

\begin{abstract}
The magnetic phase diagrams of all dilute $\mathrm{Cr}$ alloys can be explained by a simple theoretical model with coupled spin- and charge-density waves and a finite electron reservoir. If the charge-density wave and electron reservoir are sufficiently large, the paramagnetic to commensurate spin-density wave transition becomes strongly first order, as found in $\mathrm{Cr}_{1-x} \mathrm{Fe}_{x}$ and $\mathrm{Cr}_{1-x} \mathrm{Si}_{x}$ alloys. The observed discontinuity of the slope $d T_{N} / d x$ at the triple point and the bending of the $\mathrm{CI}$ phase boundary are also natural consequences of this model.
\end{abstract}

\section{DISCLAIMER}

\begin{abstract}
This report was prepared as an account of work sponsored by an agency of the United States Government. Neither the United States Government nor any agency thereof, nor any of their employees, makes any warranty, express or implied, or assumes any legal liability or responsibility for the accuracy, completeness, or usefulness of any information, apparatus, product, or process disclosed, or represents that its use would not infringe privately owned rights. Reference herein to any specific commercial product, process, or service by trade name, trademark, manufacturer, or otherwise does not necessarily constitute or imply its endorsement, recommendation, or favoring by the United States Government or any agency thereof. The views and opinions of authors expressed herein do not necessarily state or reflect those of the United States Government or any agency thereof.
\end{abstract}




\section{DISCLAIMER}

Portions of this document may be illegible in electronic image products. Images are produced from the best available original document. 
Since the spin-density wave ${ }^{1,2}$ (SDW) of $\mathrm{Cr}$ alloys is produced by the Coulomb attraction between electrons and holes on almost perfectly nested Fermi surfaces, the wavevector and amplitude of the SDW can be controlled by changing the relative sizes of those Fermi surfaces with doping. For pure $\mathrm{Cr}_{\mathrm{r}}{ }^{3}$ the SDW is incommensurate (I) with the bcc lattice and the paramagnetic $(\mathrm{P})$ to I phase transition at $310 \mathrm{~K}$ is weakly first order. When the electron Fermi surface is sufficiently close in size ${ }^{4}$ to the hole Fermi surface, the SDW becomes commensurate (C) with the lattice. In the simplest model of $\mathrm{Cr}$ alloys, dopants affect the nesting free energy solely by altering the mismatch between the Fermi surfaces. But it is well-known ${ }^{3}$ that different dopants have dramatically different effects on the magnetic ordering and phase boundaries of $\mathrm{Cr}$ alloys. Whereas the $\mathrm{PC}$ transition in other alloys is second order, the PC transition is strongly first order in $\mathrm{CrFe}$ and $\mathrm{CrSi}$ alloys. While a second-order $\mathrm{CI}$ phase boundary falls to the $\mathrm{C}$ side of the triple point for $\mathrm{CrMn}, \mathrm{CrRe}$, and $\mathrm{CrRu}$ alloys, a first-order $\mathrm{CI}$ boundary falls to the I side for $\mathrm{CrFe}$ alloys. This diversity has puzzled theorists and delighted experimentalists for many years. Here, we argue that the magnetic phase diagrams of all dilute $\mathrm{Cr}$ alloys can be qualitatively explained by a simple model with a coupled charge-density wave (CDW) and a finite reservoir of electrons.

Both $x-$ ray $^{5,6}$ and neutron scattering ${ }^{7,8}$ measurements revealed the existence of a CDW in pure $\mathrm{Cr}$ over 20 years ago. Recently, Hill et al. ${ }^{9}$ performed an exhaustive set of x-ray measurements on the temperature dependence of the CDW. If the wavevectors of the ISDW are $Q_{ \pm}^{\prime}=(G / 2)\left(1 \pm \partial^{\prime}\right)$, on either side of $G / 2=2 \pi \hat{z} / a$, then the wavevectors of the CDW are $2 \mathbf{Q}_{ \pm}^{\prime}=\mathbf{G}\left(1 \pm \partial^{\prime}\right)$, on either side of the reciprocal latrice vector $\mathbf{G}$. In the I phase, a strain wave ${ }^{10,11}$ couples to the CDW with the same wavevectors $2 Q_{ \pm}^{\prime}$. Young and Sokoloff ${ }^{12}$ suggested that the CDW was responsible for the weak first-order PI transition of pure Cr. However, its effect on the phase diagram was never considered.

The SDW consists of spin-triplet electron-hole pairs formed by the Coulomb attraction $U>0$ between electrons and holes on Fermi surfaces $a$ and $b$. To minimize the condensation free energy ${ }^{4}$ on both sides of the two Fermi surfaces, the ordering wavevectors $\mathbf{Q}_{ \pm}^{\prime}$ of the SDW lie closer to $G / 2$ than the nesting wavevectors $Q_{ \pm}=(G / 2)(1 \pm \partial)$. The mismatch 
$\partial$ between the $a$ and $b$ Fermi surfaces can be controlled by doping with another transition metal: adding $\mathrm{Mn}, \mathrm{Fe}, \mathrm{Re}$, or $\mathrm{Ru}$ raises the chemical potential and decreases $\partial$; adding $\mathrm{V}$ lowers the chemical potential and increases the mismatch $\partial$. For pure $\mathrm{Cr}, \partial \approx 0.05$ so the hole Fermi surface is only slightly larger than the electron surface. As $\partial$ decreases and the nesting improves, the SDW wavevector parameter $\partial^{\prime}<\partial$ also decreases until, for a small enough $\partial>0$, the SDW becomes commensurate with $\partial^{\prime}=0$.

The energy mismatch between the Fermi surfaces is given by $z_{0}=4 \pi \partial v_{F} / \sqrt{3} a$, which increases linearly with the $\mathrm{V}$ concentration and decreases linearly with the concentration of $\mathrm{Mn}, \mathrm{Fe}, \mathrm{Re}$, or $\mathrm{Ru}$. For pure $\mathrm{Cr}, z_{0} \approx 475 \mathrm{meV}$. Another quantity which will appear shortly is the Néel temperature $T_{N}^{*} \approx 80 \mathrm{meV}$ of a perfectly nested Cr alloy with $z_{0}=0$.

Since $z_{0}>0$, the hole Fermi surface is larger than the electron Fermi surface and not all of the $b$ holes can be paired to $a$ electrons in the SDW. The density of unpaired holes ${ }^{13}$ on the $b$ Fermi surface is given by $\rho_{e h} z_{0} / 4$. As first shown by Young and Sokoloff, ${ }^{12}$ the Coulomb attraction $U^{\prime}>0$ between unpaired holes and paired electrons on the $b$ Fermi surface produces the CDW in the I phase with order parameter $\delta \propto-U^{\prime}\left(g / T_{N}^{*}\right)^{2}<0$.

Starting with the Green's function of $\mathrm{Cr}$ alloys, we have shown ${ }^{14}$ that the spin and charge distributions are given by

$$
\begin{gathered}
\mathbf{S}(\mathbf{r})=-\frac{\hbar}{4 \lambda} V \rho_{e h} g \hat{\mathbf{m}}|u(\mathbf{r})|^{2}\left\{\cos \left(\mathbf{Q}_{+}^{\prime} \cdot \mathbf{r}-\phi_{+}\right)+\cos \left(\mathbf{Q}_{-}^{\prime} \cdot \mathbf{r}-\phi_{-}\right)\right\} \\
\varrho(\mathbf{r})=-\frac{1}{2 \lambda^{\prime}} V \rho_{e h} \delta|u(\mathbf{r})|^{2} \cos \left(\left(\mathbf{Q}_{+}^{\prime}-\mathbf{Q}_{-}^{\prime}\right) \cdot \mathbf{r}-\phi_{+}+\phi_{-}\right)
\end{gathered}
$$

where $\lambda=U \rho_{e h} / 8$ and $\lambda^{\prime}=U^{\prime} \rho_{e h} / 8$ are dimensionless coupling constants and $u(\mathbf{r})$ is a periodic Bloch function normalized to one in volume $V$. These relations imply that the SDW and CDW are in phase: the electron number is a maximum whenever the spin is a maximum or a minimum. Because the Bloch functions of the $\mathrm{d}$-band electrons are strongly peaked at the atomic sites, the amplitudes of the spin and electron number in the I phase are $S_{0}=-(\hbar g / 2 \lambda) \cos \left(\left(\phi_{+}+\phi_{-}\right) / 2\right)(V / N) \rho_{e h}$ and $\varrho_{0}=-\left(\delta / 2 \lambda^{\prime}\right)(V / N) \rho_{e h}$. Of course, the CDW $\varrho(\mathbf{r})$ carries a net charge only in the $C$ phase with $\mathbf{Q}_{+}^{\prime}=\mathbf{Q}_{-}^{\prime}$. But in order to conserve 
electron number, the CCDW must vanish and the phase difference $\phi_{+}-\phi_{-}$must equal $\pi / 2$ plus some multiple of $\pi$.

Besides the nesting electron jack and hole octahedron centered at the $\Gamma$ and $H$ points in reciprocal space, the band structure ${ }^{15}$ of $\mathrm{Cr}$ alloys also contains electron balls midway between the $\Gamma$ and $H$ points and hole pockets at the $N$ points. These other bands may be lumped together into an electron reservoir ${ }^{16.17}$ with density-of-states $\rho_{r}$. If the density-ofstates of the $a$ and $b$ bands is $\rho_{e h}$, then the power of the reservoir is defined by $p=\rho_{r} / \rho_{e h}$. Below $T_{N}$, the SDW removes electrons from the $a$ and $b$ Fermi surfaces. So for a finite reservoir with $p<\infty$, the chemical potential will decrease and the wavevector mismatch $\partial$ will increase as the SDW grows. Since jumps in the order parameters require sudden changes in the chemical potential, a finite reservoir suppresses first-order phase transitions.

The free energy difference $\Delta F(p, T)$ between the magnetic and paramagnetic states is derived elsewhere. ${ }^{14}$ When $p<\infty, z_{0}(T)$ increases with decreasing temperature and the phase diagram is evaluated in terms of the paramagnetic mismatch $z_{0}=z_{0}\left(T>T_{N}\right)$. Both order parameters $g$ and $\delta$ as well as the SDW wavevector parameter $\partial^{\prime}$ are obtained by minimizing $\Delta F(p, T)$. Although the self-consistent equations for those parameters are not affected by the finite reservoir, solutions which are saddle points or even maxima of $\Delta F(p=\infty, T)$ may become the minima of $\Delta F(p, T)$ when $p<\infty$.

Our results for an infinite reservoir are presented in Fig.1. Surprisingly, the PI transition is always second order. By equating the SDW ordering and nesting wavevectors (equivalent to setting $\partial^{\prime}=\partial$ ), Young and Sokolof ${ }^{12}$ obtained a first-order PI transition above a threshold value of $\lambda^{\prime}$. However, when the free energy $\Delta F(p, T)$ is minimized with respect to $\partial^{\prime}$, the I solutions near the first-order phase boundary are always unstable. Instead, a nonzero CDW drives a first-order PC transition and pushes the triple point towards higher values of $=0$. As $\lambda^{\prime} \rightarrow 1 / 2$, the CDW becomes unstable and the I phase disappears. For $\lambda^{\prime}>1 / 2$, the free energy is minimized when $\delta=-\infty$ and no physical solutions exist.

A natural consequence of this model is the discontinuity at the triple point in the derivative of the Néel temperature with respect to either the energy mismatch $z_{0}(x)$ or the dopant 
concentration $x$. While this discontinuity has been observed in all $\mathrm{Cr}_{r_{1-x}} \mathrm{~A}_{x}$ alloys with a triple point, ${ }^{3}$ it has never been previously explained.

The normalized and dimensionless latent heat $L^{\prime}=L / \rho_{e h} T_{N}^{* 2}$ of the first-order PC transition is plotted in the inset of Fig.1. As observed in $\mathrm{CrFe}$ and $\mathrm{CrSi}$ alloys, ${ }^{3}$ the latent heat peaks to the left of the triple point due to the rapid drop in the first-order Néel temperature $T_{N}$ near the triple point. If $\rho_{\text {eh }}=2.4$ states $/ \mathrm{Ry}$ atom, ${ }^{18}$ then the observed maximum latent heat of $12.6 \mathrm{~J} / \mathrm{mol}$ for $\mathrm{CrFe}$ alloys is fitted by $\lambda^{\prime} \approx 0.135$. Correspondingly, the CDW has an amplitude of $\varrho_{0} \approx 0.005$ electrons per site at zero temperature on the I side of the CI phase boundary.

Although it correctly predicts the strongly first-order $\mathrm{PC}$ transition in $\mathrm{CrFe}$ and $\mathrm{CrSi}$ alloys, the free energy $\Delta F(p=\infty, T)$ cannot explain the weak first-order PI transition in pure $\mathrm{Cr}$ or the second-order $\mathrm{CI}$ transition in CrMn. These deficiencies are remedied by including the effects of a finite electron reservoir.

Since the effective mismatch $z_{0}(T)$ increases with decreasing temperature, a finite electron reservoir favors the I phase of the SDW. When $p$ is sufficiently small, the full free energy $\Delta F(p, T)$ stabilizes the formerly unstable I solutions near the first-order phase boundary. Fixing $\lambda^{\prime}=0.4$, we plot the phase diagram for three powers of the reservoir in Fig.2. With decreasing reservoir power, the triple point shifts towards smaller values of $z_{0}$ and higher temperatures, the first-order Néel temperature is suppressed, and the CI phase boundary bends towards the $\mathrm{C}$ side of the triple point. For $p=0$ and $\lambda^{\prime}=0.4$, the first-order $\mathrm{PC}$ transition is replaced by a weak first-order PI transition, just as observed in CrMn alloys. For $p<2.4$ and any $\lambda^{\prime}<1 / 2$, the CI phase transition becomes second order, as observed in most $\mathrm{Cr}$ alloys. ${ }^{3}$ The inset to Fig. 2 shows that the latent heat of the first-order PC or PI phase transition is suppressed as the reservoir power decreases. So the estimates given above for $\lambda^{\prime}$ and $\varrho_{0}$ in CrFe alloys are only lower bounds.

At least qualitatively, the $\mathrm{C}$ and I phase boundaries of all dilute $\mathrm{Cr}$ alloys can be explained by the dependences of the reservoir power $p$ and the coupling $\lambda^{\prime}$ on the impurity concentration $x$. While the originally proposed model ${ }^{12}$ for the weak first-order PI transi- 
tion in pure $\mathrm{Cr}$ was flawed, our slightly more complex model with a finite reservoir produces such a phase transition. For $\mathrm{Cr} \mathrm{Mn}$ alloys, a first-order $\mathrm{PC}$ transition may be prevented by the suppression of $\lambda^{\prime}$ with the Mn concentration. But unlike other impurity atoms, ${ }^{3} \mathrm{Fe}$ impurities retain their localized magnetic moments ${ }^{19,20}$ within the $\mathrm{Cr}$ host below $T_{N}$. Since fewer of its impurity electrons enter the conduction band, the triple point for $\mathrm{Cr} F e$ alloys lies at a higher concentration than for $\mathrm{CrMn}$ alloys: $2.4 \% \mathrm{Fe}$ compared to $0.3 \% \mathrm{Mn}$. By enlarging the electron reservoir, the magnetostriction ${ }^{21}$ of $\mathrm{CrFe}$ alloys bends the first-order CI phase boundary to the I side of the triple point. By enhancing the coupling constant $\lambda^{\prime}$, the indirect exchange of $b+$ holes and $b$ - electrons mediated by the paramagnetic $\mathrm{Fe}$ moments may generate a large CDW in the I phase and drives the strong first-order PC transition of $\mathrm{CrFe}$ alloys.

Unfortunately, this picture cannot explain the strong first-order PC transition produced by nonmagnetic $\mathrm{Si}$ impurities. So the dramatic effect of $\mathrm{Si}$ on the magnetic phase boundary and re-entrant $\mathrm{C}$ phase ${ }^{3}$ of $\mathrm{CrSi}$ alloys cannot be explained by our model. Nonetheless, we believe that the strong first-order PC transition in $\mathrm{CrSi}$ alloys is also preceeded by the development of a large CDW in the I phase.

Several traits can now be associated with the magnitudes of $\lambda^{\prime}$ and $p$. Along with the strong first-order $\mathrm{PC}$ transition, large values of $\lambda^{\prime}$ and $p$ also bend the first-order $\mathrm{Cl}$ phase boundary to the I side of the triple point and generate a large discontinuity in $d T_{N} / d z_{0}$ at the triple point. All of these traits appear in $\mathrm{CrFe}$ and $\mathrm{CrSi}$ alloys. ${ }^{3}$ Small values of $\lambda^{\prime}$ and $p$, on the other hand, are associated with a second-order PC transition, a second-order CI phase boundary pushing into the $\mathrm{C}$ phase, and a smaller discontinuity in $d T_{N} / d z_{0}$. These characteristics are shared by $\mathrm{CrMn}, \mathrm{CrRe}$, and $\mathrm{CrRu}$ alloys. ${ }^{3}$

The growth of the CDW on the I side of the phase boundary should be straightforward to verify. As first observed experimentally ${ }^{8}$ and confirmed by our model, the ratio

$$
\frac{\varrho_{0}}{S_{0}^{2}} \propto-\frac{\delta(T) T_{N}^{*}}{\lambda^{\prime} g(T)^{2}} \propto \frac{1}{1-2 \lambda^{\prime}}
$$

is almost independent of temperature and provides a direct measure of the coupling constant 
$\lambda^{\prime}$. So low-temperature $x$-ray or neutron-scattering measurements of $Q_{0} / S_{0}^{2}$ on a series of I $\mathrm{Cr}_{1-x} \mathrm{~A}_{x}$ alloys can confirm that $\lambda^{\prime}(x)$ grows with the $\mathrm{Fe}$ or $\mathrm{Si}$ concentration and decreases with the Mn concentration.

The model presented in this paper is certainly not complete. Impurity scattering ${ }^{22}$ must be included to explain the linear decrease ${ }^{3}$ of the Néel temperature with the $\mathrm{V}$ concentration. Spin-orbit coupling is needed to explain the presence ${ }^{3}$ of both transversely ( $\hat{\mathbf{m}} \perp \mathbf{Q}_{ \pm}^{\prime}, T>120$ $\mathrm{K}$ ) and longitudinally ( $\hat{\mathrm{m}} \| \mathrm{Q}_{ \pm}^{\prime}, T<120 \mathrm{~K}$ ) polarized phases in pure $\mathrm{Cr}$. But we believe that our basic model, with coupled spin- and charge-density waves in the presence of a finite electron reservoir, provides the simplest qualitative explanation for the magnetic phase diagrams of all dilute $\mathrm{Cr}$ alloys.

One of us (RF) would like acknowledge support from the C.S. Department of Energy under Contract No. DE-FG06-94ER45519 and under Contract Lo. DE-AC0586OR22464 with Lockheed Martin Energy Research Corp. Useful conversations with B. Larson, V.S. Viswanath, and especially with S.H. Liu are also gratefully acknowledged. 


\section{REFERENCES}

- Current address: Center for Ceramic Research, Rutgers Lniversity, Piscataway, NJ 088550909

${ }^{1}$ W.M. Lomer, Proc. Phys. Soc. London 80, 489 (1962).

${ }^{2}$ P.A. Fedders and P.C. Martin, Phys. Rev. 143, 8245 (1966).

${ }^{3}$ The properties of $\mathrm{Cr}$ alloys are reviewed by E. Fawcett, H.L. Alberts, V. Yu. Galkin, D.R. Noakes, and J. V. Yakhmi, Rev. Mod. Phys. 66, 26 (1994). In particular, this comprehensive work contains all the references on $\mathrm{CrFe}$ and $\mathrm{CrSi}$ alloys.

${ }^{4}$ R.S. Fishman and S.H. Liu, Phys. Rev. B 48, 3820 (1993).

${ }^{5}$ Y. Tsunoda, M. Mori, N. Kunitomi, Y. Teraoka, and J. Kanamori, Solid State Commun. 14, 287 (1974).

${ }^{6}$ A.F. Prekul and S.V. Sudareva, Phys. Met. Metall. 46. 46 (1979).

${ }^{7}$ R. Pynn, W. Press, S.M. Shapiro, and S.A. Werner, Phys. Rev. B 13, 295 (1976).

${ }^{8}$ S. Iida, M. Khono, Y. Tsunoda, and N. Kunitomi, J. Phys. Soc. Jap. 50, 2581 (1981).

9 J.P. Hill, G. Hegelsen, and D. Gibbs, Phys. Rev. B 51. 10336 (1995).

${ }^{10}$ S. Nakajima and Y. Kurihara, J. Phys. Soc. Japan 38, 330 (1975).

${ }^{11}$ M. Mori and Y. Tsunoda, J. Phys. C 5, L77 (1993).

${ }^{12}$ C.Y. Young and J.B. Sokoloff, J. Phys. F 4, 1304 (1974).

${ }^{13}$ R.S. Fishman and V.S. Viswanath, (unpublished).

${ }^{14}$ X.W. Jiang and R.S. Fishman, (unpublished).

${ }^{15}$ S. Asano and J. Yamashita, J. Phys. Soc. Jap. 23, 714 (1967).

${ }^{16}$ A. Shibatani, K. Motizuki, and T. Nagamiya, Phys. Rev. 177, 984 (1969). 
${ }^{17}$ R.S. Fishman and S.H. Liu, Phys. Rev. B 49, 3308 (1994).

${ }^{18}$ H.L. Skriver, J. Phys. F: Metal Phys. 11, 97 (1981).

${ }^{19}$ Y. Ishikawa, S. Hoshino, and Y. Endoh, J. Phys. Soc. Japan 22, 1221 (1967).

${ }^{20}$ T. Suzuki, J. Phys. Soc. Japan 43, 869 (1977).

${ }^{21}$ K. Nakanishi and T. Kasuya, J. Phys. Soc. Jap. 42, 833 (1977).

22 J. Zittartz, Phys. Rev. 164, 575 (1967). 


\section{FIGURES}

FIG. 1. The phase diagram of $\mathrm{Cr}$ alloys with $\rho=\infty$ and $\lambda^{\prime}=0.4$ (solid), 0.35 (long dash), 0.30 (medium dash), and 0.20 (short dash). The triple points are labeled by the filled circles and the second order paramagnetic phase boundary is denoted by a thin solid line. Inset is the normalized latent heat $L^{\prime}=L / \rho_{e h} T_{N}^{* 2}$ for the same parameters. The three different magnetic phases are labeled.

FIG. 2. The phase diagram of $\mathrm{Cr}$ alloys with $\lambda^{\prime}=0.4$ and $\rho=\infty$ (solid), 1 (long dash), or 0 (short dash). The normalized latent heat $L^{\prime}$ is inset for the same parameters with the triple points marked. 


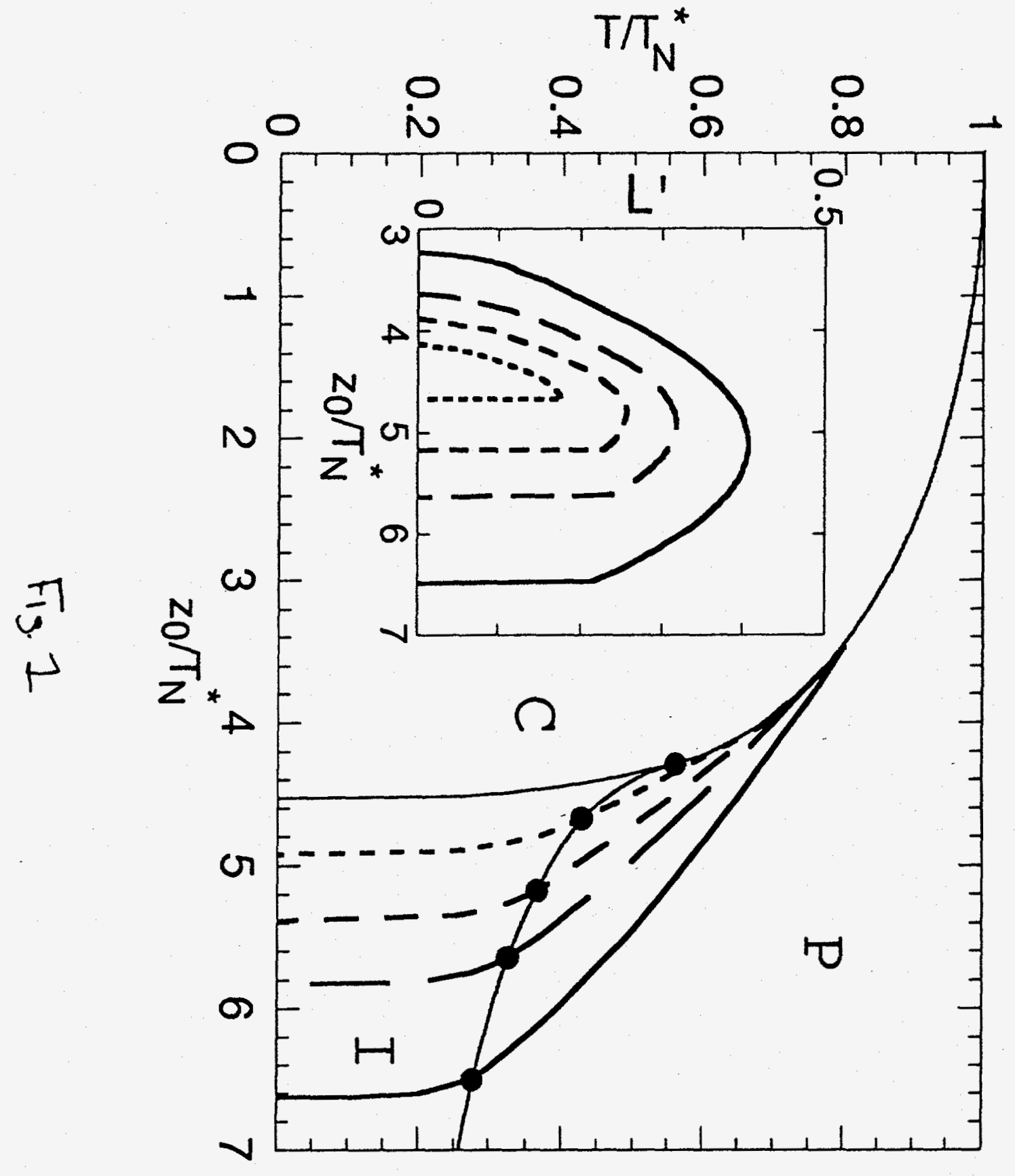




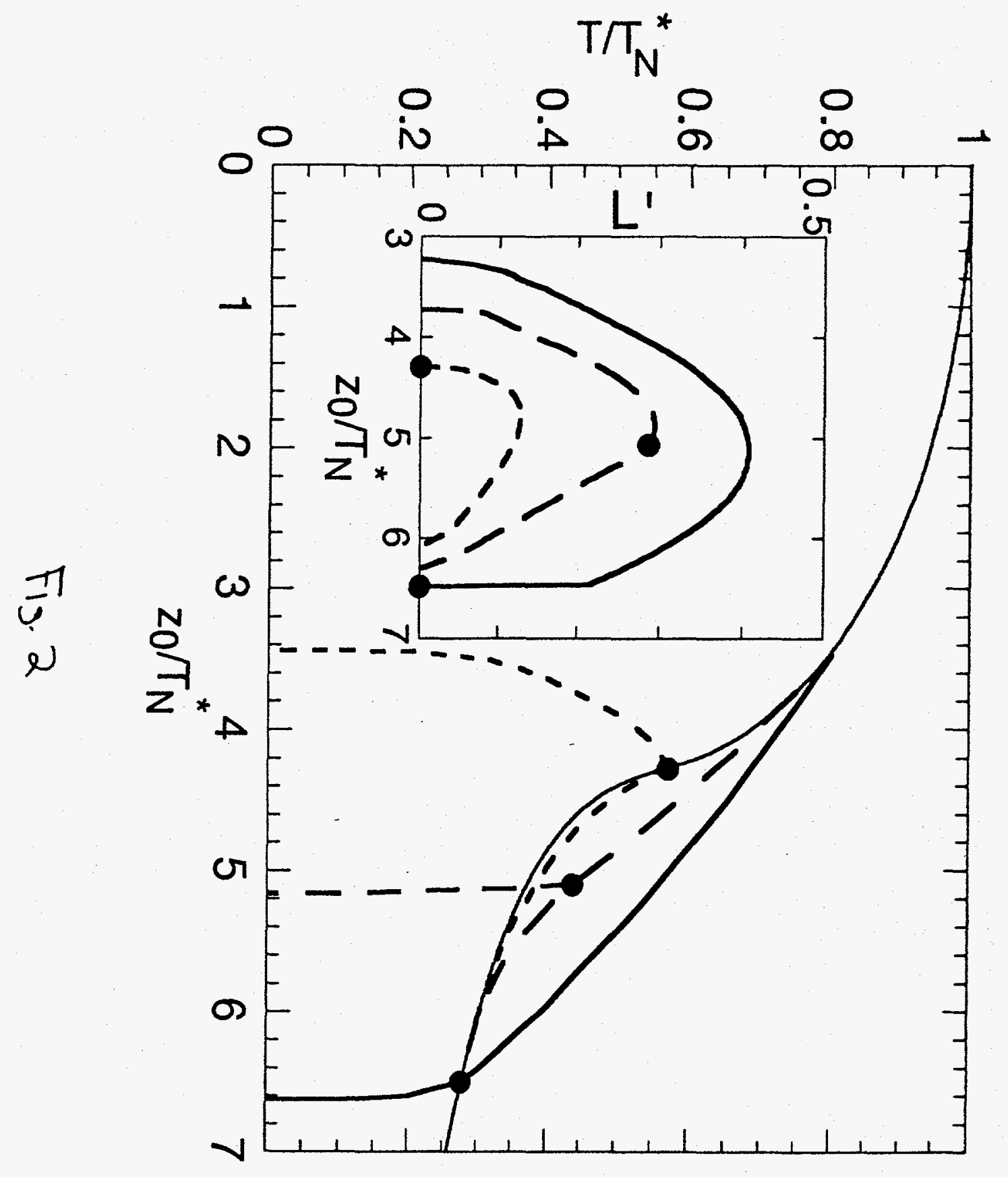

